\title{
LINEAR AUTONOMOUS NEUTRAI FUNCTIONAL DIFFERENTIAL EQUATIONS IN THE PHASE SPACE OF REGULATED FUNCTIONS
}

\author{
LUIZ FICHMANN
}

\begin{abstract}
We extend the natural description of the spectrum for the flow of the linear equation $\frac{d}{d t} D x_{t}=L x_{t}$ from the context of continuous functions to the context of regulated right-continuous functions.
\end{abstract}

\section{Introduction}

This paper intends to present a result contained in [3], namely, the extension of the spectral results of [6] for the flow of the Linear Autonomous NFDEs to the context of regulated right-continuous functions.

If $[a, b]$ is an interval of the real line and $X$ is a Banach space, we write $G([a, b], X)$ for the space of the functions $\psi:[a, b] \rightarrow X$ for which there exist the limits $\psi\left(t^{+}\right)$for every $t \in\left[a, b\left[\right.\right.$ and $\psi\left(t^{-}\right)$for every $\left.\left.t \in\right] a, b\right]$. Such functions are called regulated functions.

In [3] we extend some results obtained by J. Hale ([4]) and D. Henry ([6]) for the socalled Neutral Functional Differential Equations (NFDEs), which have the form $\frac{d}{d t}(x(t)-$ $\left.f\left(t, x_{t}\right)\right)=g\left(t, x_{t}\right)$, from the context of continuous functions to the context of regulated functions. The motivation for this extension is the fact that the fundamental matrix, which appears in the variation-of-constants formula of the linear non-homogeneous NFDE $([4],[6])$, is regulated and not continuous in $t$. So, the space of regulated functions appears as a natural context to include the fundamental matrix or the resolvent, in the case we consider a generic Banach space $X$. In this general context, Hönig ([8],[9]) studied the Volterra-Stieltjes linear Integral Equations. We applied these results, since the initial value problem of a linear NFDE leads to such an integral equation ([2]).

Another extension of the phase-space $\mathcal{C}$ of continuos functions, for which the variation -of-constants formula has a functional analytic sense, was done by Diekmann in [1] for retarded equations (NFDEs with $f \equiv 0$ ), where the author developed the theory called

Received August 5, 1992; revised April 6, 1994.

1991 Mathematics Subject Classification. 34K40.

Key words and phrases. Neutral functional equation, spectrum, regulated functions. 
"sun-star calculus". This theory consists of considering the adjoint of the flow of the linear equation in the dual space and taking the restriction to the maximal subspace where strong-continuity holds and again taking the adjoint. In this way $\mathcal{C}$ is embedded into the product space $M_{\infty}=\mathbb{R}^{n} \times L_{\infty}$, a nice space to include the fundamental matrix, and one can use the variation-of-constants formula in the weak-* sense.

Another possible approach is to choose $M_{p}=\mathbb{R}^{n} \times L_{p}$ as the phase-space. This was done in [10] for a certain class of NFDEs.

\section{The main result}

Let $\mathbb{E}^{n}$ denote the Euclidean space of real or complex $n$-vectors and let $r$ be a fixed positive number. $\mathcal{G}^{+}=G^{+}\left([-r, 0], \mathbb{E}^{n}\right)$ is the space of the regulated right-continuous functions $\varphi:[-r, 0] \rightarrow \mathbb{E}^{n}$, which is complete with the norm $\|\varphi\|=\sup _{-r \leq \theta \leq 0}\|\varphi(\theta)\|$. We call $C=C\left([-r, 0], \mathbb{E}^{n}\right)$ the closed subspace of $\mathcal{G}^{+}$of continuous functions. If $x$ is a regulated right-continuous map of $[a-r, b]$ into $\mathbb{E}^{n}$, then $x_{t} \in \mathcal{G}^{+}$is given, for each $a \leq t \leq b$, by $x_{t}(\theta)=x(t+\theta),-r \leq \theta \leq 0$.

Let $D, L$ be fixed continuous linear functionals from $\mathcal{G}^{+}$into $\mathbb{E}^{n}$, with integral representations given by $D \varphi=\varphi(0)-\int_{-r}^{0} d \mu(\theta) \varphi(\theta)$ and $L \varphi=\int_{-\tau}^{0} d \eta(\theta) \varphi(\theta)$ for $\varphi \in \mathcal{G}^{+}$; where $\mu, \eta$ are matrix-valued functions (from $[-r, 0]$ into $\mathcal{L}\left(\mathbb{E}^{n}\right)$ ) of bounded variation which vanish at $\theta=0$ and are left-continuous. For these represrentations, we utilize the Interior Integral which extends the Riemann-Stieltjes Integral (see [8]). We assume here that $\mu$ has no singular part, i.e., $\int_{-r}^{0} d \mu(\theta) \varphi(\theta)=\sum_{k=1}^{\infty} A_{k} \varphi\left(-r_{k}\right)+\int_{-\tau}^{0} A(\theta) \varphi(\theta) d \theta$, $\forall \varphi \in \mathcal{G}^{+}$, where $0 \leq r_{k} \leq r$ and $A_{k} \in \mathcal{L}\left(\mathbb{E}^{n}\right)$ for $k \in \mathbb{N}$ and $A \in L_{1}\left([-r, 0], \mathcal{L}\left(\mathbb{E}^{n}\right)\right)$.

In this situation, the initial value problem is well posed for the NFDE:

$$
\frac{d}{d t} D x_{t}=L x_{t}, \quad t \geq 0
$$

that is, for $\varphi \in \mathcal{G}^{+}$we have the unique regulated right-continuous solution $x=x(0, \varphi)$ of $(N)$ for $t \geq 0$ with $x_{0}=\varphi$. We have, then, well defined the flow of $(N),\{T(t)\}_{t \geq 0}$, semigroup of bounded linear operators on $\mathcal{G}^{+}$given by $T(t) \varphi=x_{t}(0, \varphi)$ for $\varphi \in \mathcal{G}^{+}$and $t \geq 0$.

Let $D^{0}$ be the jump part of $D$, that is, $D^{0} \varphi=\varphi(0)-\sum_{k=1}^{\infty} A_{k} \varphi\left(-r_{k}\right)$ for $\varphi \in \mathcal{G}^{+}$. We denote by $\mathcal{G}_{D^{0}}^{+}$the kernel of $D^{0}$. The initial value problem is also well posed for the difference equation $(D)_{0}: D^{0} x_{t}=0, t \geq 0$. This defines the flow of $(D)_{0},\left\{T^{0}(t)\right\}_{t \geq 0}$, semigroup of bounded linear operators on $\mathcal{G}_{D^{0}}^{+}$.

We known that $\mathcal{C}$ is invariant under $T(t)(t \geq 0)$ and $\mathcal{C}_{D^{0}} \stackrel{\text { def. }}{=} \mathcal{G}_{D^{0}}^{+} \cap \mathcal{C}$ is invariant under $T^{0}(t)(t \geq 0)$, that is, the solution of $(N)$ or $(D)_{0}$ is continuous whenever the initial data is a continuous function.

Daniel Henry ([6], [7]) gives a complete description of the spectrum of the operators $T^{0}(t)_{\mid \mathcal{C}_{D^{0}}}$ and $T(t)_{\mid C}$ for $t \geq 0$, using the infinitesimal generator $\mathbb{A}^{0}$ of $\left\{T^{0}(t)_{\mid C_{D^{0}}}\right\}_{t \geq 0}$ 
and $\mathbf{A}$ of $\left\{T(t)_{\mid c}\right\}_{t \geq 0}$. The restriction of each flow, as above, is a strongly continuous semigroup of linear operators which admits a closed infinitesimal generator with dense domain in $\mathcal{C}_{D^{0}}$ and $\mathcal{C}$, respectively. Namely $\mathcal{D}\left(\mathbb{A}^{0}\right)=\left\{\varphi \in \mathcal{C}_{D^{0}} \mid \varphi^{\prime} \in \mathcal{C}_{D^{0}}\right\}$ with $\mathbb{A}^{0} \varphi=\varphi^{\prime}$ for $\varphi \in \mathcal{D}\left(\mathbb{A}^{0}\right)$, and $\mathcal{D}(\mathbb{A})=\left\{\varphi \in \mathcal{C} \mid \varphi^{\prime} \in \mathcal{C}\right.$ and $\left.D \varphi^{\prime}=L \varphi\right\}$ with $\mathbb{A} \varphi=\varphi^{\prime}$ for $\varphi \in \mathcal{D}(\mathbb{A})$. For the spectrum of these generators, we have:

$$
\begin{aligned}
& \sigma\left(\mathbb{A}^{0}\right)=P \sigma\left(\mathbb{A}^{0}\right)=\{\lambda \in \mathbb{C} \mid \operatorname{det} H(\lambda)=0\} \\
& \sigma(\mathbb{A})=P \sigma(\mathbb{A})=\{\lambda \in \mathbb{C} \mid \operatorname{det} \Delta(\lambda)=0\}
\end{aligned}
$$

where det $H(\lambda)=0$ and det $\Delta(\lambda)=0$ are the respective characteristic equations of $(D)_{0}$ and $(N)$, i.e.:

$$
H(\lambda)=I-\sum_{k=1}^{\infty} A_{k} e^{-\lambda r_{k}}=D^{0}\left(e^{\lambda} \cdot I\right)
$$

and $\Delta(\lambda)=\lambda H(\lambda)-\lambda \int_{-r}^{0} A(\theta) e^{\lambda \theta} d \theta-\int_{-r}^{0} d \eta(\theta) e^{\lambda \theta}=\lambda D\left(e^{\lambda \cdot I}\right)-L\left(e^{\lambda \cdot I}\right)$. Henry shows that:

$$
\sigma\left(T^{0}(t) \mid c_{D^{0}}\right) \backslash\{0\}=\overline{e^{t \sigma\left(\mathbf{A}^{0}\right)} \backslash\{0\}} \text { a.e. in } t \geq 0
$$

and $T(t)_{\mid \mathcal{C}}-T^{0}(t) \circ \Psi_{\mid \mathcal{C}}: \mathcal{C} \rightarrow \mathcal{C}$ is a compact operator for each $t \geq 0$, (where the map $\Psi$ above is a continuous projection from $\mathcal{G}^{+}$onto $\mathcal{G}_{D^{0}}^{+}$such that $\Psi(\mathcal{C}) \subset \mathcal{C}_{D^{0}}$, defined in the next section), and with these facts he concludes that:

$$
\sigma\left(T(t)_{\mid C}\right) \backslash\{0\}=\overline{e^{t \sigma(A)}} \backslash\{0\} \quad \text { a.e. in } t \geq 0 .
$$

The flows of $(D)_{0}$ and $(N)$ are neither strongly continuous nor something like "strongly regulated", for if we have a jump $T(t) \varphi(\theta)-T(t) \varphi\left(\theta^{-}\right)=2 l$, with $\|l\|>0$, for some $t \geq 0$ and $\theta \in]-r, 0]$, then $\left\|T\left(t+\epsilon_{1}\right) \varphi-T\left(t+\epsilon_{2}\right) \varphi\right\|>\|l\|$ for any $\epsilon_{1} \neq \epsilon_{2}$ in $] 0, \delta[$, for some small $\delta>0$. Then, we cannot extend the infinitesimal generators to dense domains in $\mathcal{G}_{D^{0}}^{+}$and $\mathcal{G}^{+}$respectively. Nevertheless, we still can show that the results obtained by Henry are extensible for $\mathcal{G}_{D^{0}}^{+}$and $\mathcal{G}^{+}$respectively. This is done in the next sections.

\section{The difference equation}

Let $\mathcal{E}^{+} \subset \mathcal{G}^{+}$be the space of step-functions, that is:

$\mathcal{E}^{+}=\left\{\varphi \in \mathcal{G}^{+} \mid \varphi=\sum_{i=1}^{k} c_{i} \chi_{\left[\theta_{i}, 0\right]}\right.$ for some $k \in \mathbb{N}^{*}, c_{i} \in \mathbb{E}^{n}$ and $\left.-r \leq \theta_{i} \leq 0, i=1,2, \cdots, k,\right\}$ where, for $J \subset[-r, 0], \chi_{J}(\theta)=1$ if $\theta \in J$ and $\chi_{J}(\theta)=0$ if $\theta \notin J$.

$\mathcal{E}^{+}$is a dense subspace of $\mathcal{G}^{+}$(see [8]).

Let $G^{-} B V_{0}=G^{-} B V_{0}\left([-r, 0],\left(\mathbb{E}^{n}\right)^{\prime}\right)$ be the space of applications $\alpha:[-r, 0] \rightarrow$ $\left(\mathbb{E}^{n}\right)^{\prime}=\mathcal{L}\left(\mathbb{E}^{n}, \mathbb{E}\right)$ with bounded variation which vanish at $\theta=0$ and are left-continuous. 
We have the following immediate lemmas:

Lemma. 1. For each $\varphi \in \mathcal{E}^{+}$, there is a sequence $\left\{\varphi_{m}\right\}_{m \in \mathbb{N}}, \varphi_{m} \in \mathcal{C}$, such that $\varphi_{m}(0)=\varphi(0),\left\|\varphi_{m}\right\|=\|\varphi\| \forall m \in \mathbb{N}$ and

$$
\int_{-r}^{0} d \alpha(\theta) \varphi_{m}(\theta) \stackrel{m \rightarrow \infty}{\longrightarrow} \int_{-r}^{0} d \alpha(\theta) \varphi(\theta) \quad \forall \alpha \in G^{-} B V_{0} .
$$

Proof. 1) Suppose first $n=1$ and $\varphi=\chi_{[\theta, 0]} \in \mathcal{E}^{+},-r \leq \theta \leq 0$. If $\theta=-r$ take $\varphi_{m}=\varphi \equiv 1$ and if $\theta \neq-r$, for $m>\frac{1}{r+\theta}$ take

$$
\varphi_{m}(\beta)=\chi_{[\theta, 0]}^{(m)}(\beta) \stackrel{\text { def. }}{=} \begin{cases}0 & \text { if }-r \leq \beta \leq \theta-\frac{1}{m} \\ m\left(\beta-\theta+\frac{1}{m}\right) & \text { if } \theta-\frac{1}{m} \leq \beta \leq \theta \\ 1 & \text { if } \theta \leq \beta \leq 0 .\end{cases}
$$

Then, for $\alpha \in G^{-} B \dot{V}_{0}$ we have

$$
\begin{aligned}
\int_{-r}^{0} d \alpha(\beta) \chi_{[\theta, 0]}^{(m)}(\beta) & =\int_{\theta-\frac{1}{m}}^{\theta} d \alpha(\beta)[m(\beta-\theta)]-\alpha\left(\theta-\frac{1}{m}\right) \\
& \stackrel{m \rightarrow \infty}{\longrightarrow}-\alpha(\theta)=\int_{-r}^{0} d \alpha(\beta) \chi_{[\theta, 0]}(\beta) \\
& \text { since }\left|\int_{\theta-\frac{1}{m}}^{\theta} d \alpha(\beta)[m(\beta-\theta)]\right| \leq \operatorname{Var}_{\left[\theta-\frac{1}{m}, \theta\right]}[\alpha] \stackrel{m \rightarrow \infty}{\longrightarrow} 0 .
\end{aligned}
$$

2) For the general case, we remember first that, for $\alpha \in G^{-} B V_{0}$, we have the scalar functions $\alpha_{j} \in G^{-} B V_{0}([-r, 0], \mathbb{E}), j=1,2, \cdots, n$, such that for each $p=\left(p_{1}, \cdots, p_{n}\right) \in$ $\mathbb{E}^{n}$ and $\theta \in[-r, 0]$ we have $\alpha(\theta) p=\sum_{j=1}^{n} \alpha_{j}(\theta) p_{j}$, and for $\varphi \in \mathcal{G}^{+}, \varphi(\theta)=$ $\left(\varphi_{1}(\theta), \cdots, \varphi_{n}(\theta)\right) \in \mathbb{E}^{n}$, we also have $\int_{-r}^{0} d \alpha(\theta) \varphi(\theta)=\sum_{j=1}^{n} \int_{-r}^{0} d \alpha_{j}(\theta) \varphi_{j}(\theta)$.

So, for $\varphi=\sum_{i=1}^{k} c_{i} \chi_{[\theta, 0]}$ in $\mathcal{E}^{+}, c_{i}=\left(c_{i}^{1}, c_{i}^{2}, \cdots, c_{i}^{n}\right) \in \mathbb{E}^{n}$, we can take $\varphi_{m}=$ $\sum_{i=1}^{k} c_{i} \chi_{\left[\theta_{i}, 0\right]}^{(m)}$ in $C$.

Then, for $\alpha \in G^{-} B V_{0}$ we have

$$
\begin{aligned}
& \int_{-\tau}^{0} d \alpha(\beta) \varphi_{m}(\beta)=\sum_{j=1}^{n} \sum_{i=1}^{k} c_{i}^{j} \int_{-r}^{0} d \alpha_{j}(\beta) \chi_{\left[\theta_{i}, 0\right]}^{(m)}(\beta) \\
& \stackrel{m \rightarrow \infty}{\longrightarrow} \sum_{j=1}^{n} \sum_{i=1}^{k} c_{i}^{j} \int_{-r}^{0} d \alpha_{j}(\beta) \chi_{\left[\theta_{i}, 0\right]}(\beta)=\int_{-r}^{0} d \alpha(\beta) \varphi(\beta) \quad \text { (by item 1)) }
\end{aligned}
$$

We see that for the $\varphi_{m}$ above we have $\varphi_{m}(0)=\varphi(0)$ and $\left\|\varphi_{m}\right\|=\|\varphi\| \forall m \in \mathbb{N}$

Lemma 2. For $\varphi \in \mathcal{G}^{+}$, if $\int_{-r}^{0} d \alpha(\beta) \varphi(\beta)=0 \forall \alpha \in G^{-} B V_{0}$, then $\varphi(\theta)=0$ for $-r \leq \theta<0$. 
Proof. As in lemma 1, we can suppose, without loss of generality, that $n=1$.

If we have for some $\theta \neq 0$ that $\varphi(\theta) \neq 0$, then $\exists \delta>0$ such that $\varphi(\theta+t) \neq 0$ for $0 \leq t \leq \delta$. Take $\alpha=\chi_{\left[-r, \theta+\frac{\delta}{2}\right]}$ and we will have $\int_{-r}^{0} d \alpha(\beta) \varphi(\beta)=-\varphi\left(\theta+\frac{\delta^{+}}{2}\right) \neq 0$, which is a contradiction.

For a linear operator $L$, we denote by $\mathcal{N}(L)$ and $\mathcal{R}(L)$ the kernel and the range, respectively.

Remark 1. In [4], ch. 12.3 , there is given a continuous projection $\Psi: \mathcal{C} \rightarrow \mathcal{C}_{D_{0}}$ such that $\Psi=I_{\mathcal{C}}-\Phi D^{0}$ where $\Psi=\left(\phi_{1}, \cdots, \phi_{n}\right), \phi_{i} \in \mathcal{C}$, satisfies $D^{0} \Phi=I, I$ is the $n \times n$-identity matrix, and $I_{\mathcal{C}}$ is the identity of $\mathcal{C}$.

So, $\mathcal{C}=\mathcal{C}_{D^{0}} \oplus \mathcal{N}(\Psi)$ and $\operatorname{dim} \mathcal{N}(\Psi)=n$ because $\mathcal{N}(\Psi)=\mathcal{R}\left(\Phi D^{0}\right)$ has $\Phi=$ $\left(\phi_{1}, \cdots, \phi_{n}\right)$ as a basis. Putting $\varphi^{0}=\Psi \varphi$, we have, for $\varphi \in \mathcal{C}$ :

$$
\varphi=\varphi^{0}+\Phi D^{0} \varphi=\varphi^{0}+\sum_{i=1}^{n}\left(D^{0} \varphi\right)_{i} \phi_{i}=\varphi^{0}+\sum_{i=1}^{n}\left(\varphi(0)_{i}-\int_{-r}^{0} d \bar{\mu}_{i}(\theta) \varphi(\theta)\right) \phi_{i}
$$

where $\left(D^{0} \varphi\right)_{i}$ is the $i$-th component of the vector $D^{0} \varphi \in \mathbb{E}^{n}$ and $\bar{\mu}_{i}(\theta)$ is the $i$-th line of the matrix $\bar{\mu}(\theta)=-\sum_{k=1}^{\infty} A_{k} \chi_{\left.]-\infty,-r_{k}\right]}(\theta)$. Thus, $\bar{\mu}_{i} \in G^{-} B V_{0}, i=1,2, \cdots, n$.

For $\varphi \in \mathcal{G}^{+}$it is also true that $D^{0}\left(\varphi-\Phi D^{0} \varphi\right)=D^{0} \varphi-D^{0} \Phi D^{0} \varphi=0$. Therefore we can extend $\Psi: \mathcal{G}^{+} \rightarrow \mathcal{G}_{D^{0}}^{+}$as $\Psi=I_{\mathcal{G}^{+}}-\Phi D^{0}, I_{\mathcal{G}^{+}}$being the identity of $\mathcal{G}^{+}$, and we will have $\mathcal{G}^{+}=\mathcal{G}_{D^{0}}^{+} \oplus \mathcal{N}(\Psi)$, where the kernel $\mathcal{N}(\Psi)$ remains the same $n$-dimensional subspace of $\mathcal{C}$, that is, $\mathcal{N}(\Psi)=\mathcal{R}\left(\Phi D^{0}\right)$.

From this remark and lemma 1 , it follows easily the:

Lemma 3. For $\varphi \in \mathcal{E}^{+}$, let $\varphi_{m} \in \mathcal{C}, m \in \mathbb{N}$, as in lemma 1. Then

$$
\int_{-r}^{0} d \alpha(\beta) \varphi_{m}^{0}(\beta) \stackrel{m \rightarrow \infty}{\longrightarrow} \int_{-r}^{0} d \alpha(\beta) \varphi^{0}(\beta) \quad \forall \alpha \in G^{-} B V_{0}
$$

where $\varphi^{0}=\Psi \varphi$ as in remark 1 . We also have $\varphi_{m}^{0}(0) \stackrel{m \rightarrow \infty}{\longrightarrow} \varphi^{0}(0)$.

Proof. As in remark 1,

$$
\varphi_{m}^{0}=\varphi_{m}-\sum_{i=1}^{n}\left(\varphi_{m}(0)_{i}-\int_{-r}^{0} d \bar{\mu}_{i}(\theta) \varphi_{m}(\theta)\right) \phi_{i}
$$

Then, for $\alpha \in G^{-} B V_{0}$,

$$
\int_{-r}^{0} d \alpha(\beta) \varphi_{m}^{0}(\beta)=\int_{-r}^{0} d \alpha(\beta) \varphi_{m}(\beta)-\sum_{i=1}^{n}\left(\varphi_{m}(0)_{i}-\int_{-r}^{0} d \bar{\mu}_{i}(\theta) \varphi_{m}(\theta)\right) \int_{-r}^{0} d \alpha(\beta) \phi_{i}(\beta) .
$$

Considering that $\varphi_{m}(0)_{i}=\varphi(0)_{i}$, and $\bar{\mu}_{i} \in G^{-} B V_{0}$ and using lemma 1 , we have:

$$
\int_{-r}^{0} d \alpha(\beta) \varphi_{m}^{0}(\beta) \stackrel{m \rightarrow \infty}{\longrightarrow} \int_{-r}^{0} d \alpha(\beta) \varphi^{0}(\beta)
$$


and also

$$
\begin{aligned}
& \varphi_{m}^{0}(0)=\varphi_{m}(0)-\sum_{i=1}^{n}\left(\varphi_{m}(0)_{i}-\int_{-r}^{0} d \bar{\mu}_{i}(\theta) \varphi_{m}(\theta)\right) \phi_{i}(0) \\
= & \varphi(0)-\sum_{i=1}^{n}\left(\varphi(0)_{i}-\int_{-r}^{0} d \bar{\mu}_{i}(\theta) \varphi_{m}(\theta)\right) \phi_{i}(0) \stackrel{m \rightarrow \infty}{\longrightarrow} \varphi^{0}(0)
\end{aligned}
$$

Note that $\mathcal{E}_{D^{0}}^{+\stackrel{\text { def }}{=}} \Psi\left(\mathcal{E}^{+}\right)$is dense in $\mathcal{G}_{D^{0}}^{+}$, since $\mathcal{E}^{+}$is dense in $\mathcal{G}^{+}$(see [8]) and $\Psi$ is continuous from $\mathcal{G}^{+}$onto $\mathcal{G}_{D^{0}}^{+}=\Psi\left(\mathcal{G}^{+}\right)$.

Lemma 4. If $T \in \mathcal{L}\left(\mathcal{G}_{D^{0}}^{+}, \mathcal{G}^{+}\right)$with $(T \varphi)(\theta)=\int_{-\bar{r}}^{0} d_{\beta} K(\theta-\beta) \varphi(\beta)$, where we have $-r \leq-\bar{r}<0$ and $K:[-r, \bar{r}] \rightarrow \mathcal{L}\left(\mathbb{E}^{n}\right)$ has bounded variation and is right-continuous; then, for each $\alpha \in G^{-} B V_{0}$, there is a $\tilde{\alpha} \in G^{-} B V_{0}$, such that:

$$
\int_{-r}^{0} d \alpha(\theta)(T \varphi)(\theta)=\int_{-r}^{0} d \widetilde{\alpha}(\beta) \varphi(\beta) \quad \forall \varphi \in \mathcal{G}_{D^{0}}^{+}
$$

Proof. We use the theorem 2.4 of [9], which says that:

$$
\int_{-r}^{0} d \alpha(\theta) \int_{-\bar{r}}^{0} d_{\beta} K(\theta-\beta) \varphi(\beta)=\int_{-\bar{r}}^{0} d_{\beta}\left[\int_{-r}^{0} d \alpha(\theta) K(\theta-\beta)\right] \varphi(\beta)
$$

to construct a suitable $\widetilde{\alpha} \in G^{-} B V_{0}$.

Remark 2. In [3] we show that the variation-of-constants formula for the linear NFDEs ([4], [6]) remains the same in the context of regulated functions. For $\varphi \in \mathcal{G}_{D^{0}}^{+}$, the solution $y$ for $(D)_{0}$, for $t \geq 0$, with $y_{0}=\varphi$ is given by

$$
y(t)=-\sum_{k=1}^{\infty} \int_{-r_{k}}^{0} d_{\beta} X\left(t-\beta-r_{k}\right) A_{k} \varphi(\beta), \quad t \geq 0,
$$

where $X$ is the fundamental matrix given by the conditions $D^{0} X_{t}=I$ for $t \geq 0, X(0)=I$ and $X(t) \equiv 0$ for $t<0$.

We have, by [6], lemma 3.5, the following result: if $\alpha \in \mathbb{R}$ is such that $\operatorname{det} H(\lambda) \neq 0$ in some strip $|\operatorname{Re} \lambda-\alpha| \leq \delta, \delta>0$, then we may decompose $X(t)=X^{P}(t)+X^{Q}(t)$ (if $\alpha=0$ we will have $X(t)=X^{P}(t)+X^{Q}(t)+$ constant $), X^{P}$ can be extended for $t \leq 0$ and we have the estimates:

$$
\begin{aligned}
& \operatorname{Var}_{[t-r, t]}\left[X^{Q}\right] \leq M e^{(\alpha-\delta) t} \quad \text { for } t \geq 0 \\
& \operatorname{Var}_{[t-r, t]}\left[X^{P}\right] \leq M e^{(\alpha+\delta) t} \quad \text { for } t \geq 0
\end{aligned}
$$

for some constant $M$. 
Theorem 1. Suppose $\alpha \in \mathbb{R}$ is such that det $H(\lambda) \neq 0$ in some strip $\mid$ Re $\lambda-$ $\alpha \mid \leq \delta, \delta>0$. Then there exist closed subspaces $P, Q$ of $\mathcal{G}_{D^{0}}^{+}$, invariant under $T^{0}(t), t \geq 0$, such that $\mathcal{G}_{D^{0}}^{+}=P \oplus Q$, where $\oplus$ means the direct sum. The semigroup $\left\{T^{0}(t)_{\mid P}\right\}_{t \geq 0}$ may be extended uniquely as a group $(-\infty<t<\infty)$ of operators on $P$. There exists a constant $M^{\prime}$ such that : $\left\|T^{0}(t)_{\mid Q}\right\| \leq M^{\prime} . e^{(\alpha-\delta) t}$ for $t \geq 0$ and $\left\|T^{0}(t)_{\mid P}\right\| \leq M^{\prime} \cdot e^{(\alpha+\delta) t}$ for $t \leq 0$.

Proof. As in remark 2, we have the split of matrix $X$ and, for $\varphi \in \mathcal{G}_{D^{0}}^{+}$, we have the solution of $(D)_{0}, y(t)$, given by formula $\left(\rho_{D^{0}}\right)$. We may write: $y(t)=y^{P}(t)+y^{Q}(t)$, where

$$
y^{P, Q}(t) \stackrel{\text { def. }}{=}-\sum_{k=1}^{\infty} \int_{-r_{k}}^{0} d_{\beta} X^{P, Q}\left(t-\beta-r_{k}\right) A_{k} \varphi(\beta), \quad t \geq 0
$$

and we can take $-\infty<t<\infty$ for $y^{P}$. Since $X^{P}: \mathbb{R} \rightarrow \mathcal{L}\left(\mathbb{E}^{n}\right)$ and $X^{Q}: \mathbb{R}_{+} \rightarrow \mathcal{L}\left(\mathbb{E}^{n}\right)$ are right-continuous and of bounded variation in each compact interval of $t$ (see [6] lemma 3.5 ), we have that $y^{P, Q}$ are well defined and are right-continuous regulated functions because: I) each Interior Integral $\int_{-r_{k}}^{0} d_{\beta} \cdots$ in the formula defines a right-continuous regulated function of $t$ (see [9] $\left\{2\right.$ ), so $y_{N}^{P, Q}(t) \stackrel{\text { def. }}{=}-\sum_{k=1}^{N} \int_{-r_{k}}^{0} d_{\beta} X^{P, Q}\left(t-\beta-r_{k}\right) A_{k} \varphi(\beta)$ is also right-continuous regulated in $t$, for each $N \in \mathbb{N}$, and II) $\left\{y_{N}^{P}\right\}_{N \in \mathbb{N}}$ and $\left\{y_{N}^{Q}\right\}_{N \in N}$ are Cauchy-sequences in the space of right-continuous regulated functions when we take $t$ in any compact interval, with the uniform-norm, since $\sum_{k=1}^{\infty}\left|A_{k}\right|<\infty$, and then they converge as these spaces are complete (see [8]).

From remark 2, we also obtain the estimates:

$$
\begin{cases}\left\|y^{Q}(t)\right\| \leq\left(\sum_{k=1}^{\infty}\left|A_{k}\right|\right) \cdot M \cdot e^{(\alpha-\delta) t}\|\varphi\| & \text { for } t \geq 0 \\ \left\|y^{P}(t)\right\| \leq\left(\sum_{k=1}^{\infty}\left|A_{k}\right|\right) \cdot M \cdot e^{(\alpha+\delta) t}\|\varphi\| & \text { for } t \leq 0\end{cases}
$$

Define $T^{0}(t)^{P} \varphi=y_{t}^{P}$ for $t \in \mathbb{R}$ and $T^{0}(t)^{Q} \varphi=y_{t}^{Q}$ for $t \geq r$. By the majorations above we see that $T^{0}(t)^{P, Q} \in \mathcal{L}\left(\mathcal{G}_{D^{0}}^{+}, \mathcal{G}^{+}\right)$. Let $\pi_{P} \stackrel{\text { def. }}{=} T^{0}(0)^{P} \in \mathcal{L}\left(\mathcal{G}_{D^{0}}^{+}, \mathcal{G}^{+}\right)$.

In [6] theorem 3.1, it is shown that $\pi_{P} \mid \mathcal{C}_{D^{0}} \in \mathcal{L}\left(\mathcal{C}_{D^{0}}\right)$ and it is idempotent.

We will show that $\pi_{P} \in \mathcal{L}\left(\mathcal{G}_{D^{0}}^{+}\right)$and is also idempotent.

We begin with the step-functions. Let $\varphi \in \mathcal{E}^{+}$and $\varphi_{m} \in \mathcal{C}, m \in \mathbb{N}$, as in lemma 1. By remark 1, we have: $\varphi=\varphi^{0}+\sum_{i=1}^{n}\left(\varphi(0)_{i}-\int_{-r}^{0} d \bar{\mu}_{i}(\theta) \varphi(\theta)\right) \phi_{i}$. Using the formula of $\pi_{P} \varphi^{0}=y_{0}^{P}$ (now $y$ is the solution of $(D)_{0}$ with initial value $\varphi^{0}$ ) and lemmas 3 and 4 we obtain $\int_{-r}^{0} d \alpha(\beta) \pi_{P} \varphi_{m}^{0}(\beta) \stackrel{m \rightarrow \infty}{\longrightarrow} \int_{-r}^{0} d \alpha(\beta) \pi_{P} \varphi^{0}(\beta) \forall \alpha \in G^{-} B V_{0}$. By the formula of $\pi_{P} \varphi_{m}^{0}(\theta)$, for each $\theta \in[-r, 0]$, and the fact that $\left\|\varphi_{m}\right\|=\|\varphi\|$ (and then $\left\|\varphi_{m}^{0}\right\| \leq\|\Psi\| \cdot\|\varphi\|$ $\left.\forall_{m} \in \mathbb{N}\right)$, we obtain $\pi_{P} \varphi_{m}^{0}(\theta) \stackrel{m \rightarrow \infty}{\longrightarrow} \pi_{P} \varphi^{0}(\theta)$ and, in particular, $\pi_{P} \varphi_{m}^{0}(0) \stackrel{m \rightarrow \infty}{\longrightarrow} \pi_{P} \varphi^{0}(0)$. Since $\varphi_{m}^{0}=\Psi\left(\varphi_{m}\right) \in \mathcal{C}_{D^{0}}$, we have, by [6] theorem 3.1, that $\pi_{P} \varphi_{m}^{0} \in \mathcal{C}_{D^{0}}$, so $0=$ $D^{0}\left(\pi_{P} \varphi_{m}^{0}\right)=\pi_{P} \varphi_{m}^{0}(0)-\int_{-r}^{0} d \bar{\mu}(\beta)\left(\pi_{P} \varphi_{m}^{0}\right)(\beta)^{m \rightarrow \infty} \pi_{P} \varphi^{0}(0)-\int_{-r}^{0} d \bar{\mu}(\beta)\left(\pi_{P} \varphi^{0}\right)(\beta)=$ $D^{0}\left(\pi_{P} \varphi^{0}\right)$. Then $\pi_{P} \varphi^{0} \in \mathcal{G}_{D^{0}}^{+}$, that is, $\pi_{P}\left(\mathcal{E}_{D^{0}}^{+}\right) \subset \mathcal{G}_{D^{0}}^{+}$and taking the closure of $\mathcal{E}_{D^{0}}^{+}$, 
we have $\pi_{P} \in \mathcal{L}\left(\mathcal{G}_{D^{0}}^{+}\right)$. Now it makes sense to take $\pi_{P}^{2}$. To show that $\pi_{P}$ is a projection, we note first that $\int_{-r}^{0} d \alpha(\beta)\left(\pi_{P}^{2} \varphi_{m}^{0}\right)(\beta) \stackrel{m \rightarrow \infty}{\longrightarrow} \int_{-r}^{0} d \alpha(\beta)\left(\pi_{P}^{2} \varphi^{0}\right)(\beta), \forall \alpha \in G^{-} B V_{0}$, a consequence of the formula of $\pi_{P}^{2} \varphi^{0}(\beta)$ and lemma 4 . Note that

$$
\begin{aligned}
\pi_{P}^{2} \varphi^{0}(\beta)= & \left(T^{0}(0)^{P}\left[T^{0}(0)^{P} \varphi^{0}\right]\right)(\beta)=-\sum_{k=1}^{\infty} \int_{-r_{k}}^{0} d_{\tau} X^{P}\left(\beta-\tau-r_{k}\right) A_{k}\left[y^{P}(\tau)\right]= \\
& -\sum_{k=1}^{\infty} \int_{-r_{k}}^{0} d \tau X^{P}\left(\beta-\tau-r_{k}\right) A_{k}\left[-\sum_{j=1}^{\infty} \int_{-r_{j}}^{0} d_{\sigma} X^{P}\left(\tau-\sigma-r_{j}\right) A_{j} \varphi^{0}(\sigma)\right] .
\end{aligned}
$$

We know that $\pi_{P}^{2} \varphi_{m}^{0}=\pi_{P} \varphi_{m}^{0}$, since $\varphi_{m}^{0} \in \mathcal{C}_{D^{0}}$. Therefore, $\int_{-r}^{0} d \alpha(\beta)\left[\pi_{P}^{2}-\pi_{P}\right] \varphi^{0}(\beta)=0$ $\forall \alpha \in G^{-} B V_{0}$ and from lemma 2 we have $\pi_{P}^{2} \varphi^{0}(\theta)=\pi_{P} \varphi^{0}(\theta)$ for $-r \leq \theta<0$. For $\theta=0$, observe that $D^{0}\left(\pi_{P}^{2} \varphi^{0}\right)=\pi_{P}^{2} \varphi^{0}(0)-\int_{-r}^{0} d \bar{\mu}(\theta) \pi_{P}^{2} \varphi^{0}(\theta)=\pi_{P}^{2} \varphi^{0}(0)-\pi_{P} \varphi^{0}(0)+D^{0}\left(\pi_{P} \varphi^{0}\right)$, but $D^{0}\left(\pi_{P}^{2} \varphi^{0}\right)=D^{0}\left(\pi_{P} \varphi^{0}\right)=0$ since $\pi_{P} \in \mathcal{L}\left(\mathcal{G}_{D^{0}}^{+}\right)$, that is, $\pi_{P}\left(\mathcal{G}_{D^{0}}^{+}\right) \subset \mathcal{G}_{D^{0}}^{+}=\mathcal{N}\left(D^{0}\right)$. This completes the proof that $\pi_{P}$ is idempotent in $\mathcal{E}_{D^{0}}^{+}$and so in $\mathcal{G}_{D^{0}}^{+}$.

Then we have the closed subspaces of $\mathcal{G}_{D^{0}}^{+}: P=\mathcal{R}\left(\pi_{P}\right), Q=\mathcal{N}\left(\pi_{P}\right) ; \mathcal{G}_{D^{0}}^{+}=P \oplus Q$ and $\pi_{P}$ is a projection on $P$ along $Q$.

By [6] theorem 3.1, we have $T^{0}(t) \pi_{P} \varphi_{m}^{0}(\theta)=\pi_{P} T^{0}(t) \varphi_{m}^{0}(\theta)=T^{0}(t)^{P} \varphi_{m}^{0}(\theta) \forall t \geq 0$, $\forall \theta \in[-r, 0]$ and each of these expressions converges when $m \rightarrow \infty$ to the respective expression with $\varphi^{0}$ instead of $\varphi_{m}^{0}$ (this can be shown by using the formulas of $\pi_{P}, T^{0}(t), T^{0}(t)^{P}$ and lemma 4). Therefore, $T^{0}(t) \pi_{P}=\pi_{P} T^{0}(t)=T^{0}(t)^{P}$ in $\mathcal{E}_{D^{0}}^{+}$and in $\mathcal{G}_{D^{0}}^{+}$, for $t \geq 0$. For $t \in \mathbb{R}$, we also obtain $0=D^{0}\left(T^{0}(t)^{P} \varphi_{m}^{0}\right)^{m \rightarrow \infty} \longrightarrow D^{0}\left(T^{0}(t)^{P} \varphi^{0}\right)$ and this allows us to define $T^{0}(t)=T^{0}(t)^{P}$ in $P$ for $t \leq 0$ and to obtain the group of isomorphisms $\left\{T^{0}(t)_{\mid P}\right\}_{t \in \mathbb{R}}$; for, when we have the backward continuation of the solution of equations like $(D)_{0}$ in the whole line, this continuaiton is unique (see [5]).

The inequalities stated in the theorem follow immediately from inequalities in (*).

Remark 3. The subspaces $P \cap C_{D^{0}}$ and $Q \cap \mathcal{C}_{D^{0}}$ are characterized in [6] theorem 3.1, in terms of generalized eigenspaces corresponding to the eigenvalues of the infinitesimal generator $\mathbf{A}^{0}$ which have the real parts bigger than $\alpha$ and smaller than $\alpha$, respectively. We can extend, now, the theorem 3.2 of [6],

Theorem 2. Assume that $\lambda \mapsto \operatorname{det} H(\lambda)$ has zeros; then, for $t \geq 0$, we have:

$$
\overline{\left\{e^{\lambda t} \mid \operatorname{det} H(\lambda)=0\right\}} \subset \sigma\left(T^{0}(t)\right) \subset\left\{\mu|| \mu \mid=e^{\xi t}, \xi \in \overline{\mathcal{Z}}\right\} \cup\{0\},
$$

where $\mathcal{Z} \stackrel{\text { def. }}{=}\{\operatorname{Re} \lambda \mid \operatorname{det} H(\lambda)=0\}$ and $\overline{\mathcal{Z}}$ is the closure of $\mathcal{Z}$.

If $\alpha \notin \overline{\mathcal{Z}}$ and $\mathcal{G}_{D^{0}}^{+}=P \oplus Q$ is the decomposition given by theorem 1 , then

$$
\begin{aligned}
& \sigma\left(T^{0}(t)_{\mid P}\right) \subset\left\{\mu|| \mu \mid=e^{\xi t}, \xi \in \overline{\mathcal{Z}} \text { and } \xi>\alpha\right\} \\
& \sigma\left(T^{0}(t)_{\mid Q}\right) \subset\left\{\mu|| \mu \mid=e^{\xi t}, \xi \in \overline{\mathcal{Z}} \text { and } \xi<\alpha\right\}
\end{aligned}
$$

both for $t \geq 0$. 
If $\mathcal{Z}$ is empty, then $\sigma\left(T^{0}(t)\right)=\{0\}$ for $t>0$; in fact, $T^{0}(t)=0$ for $t \geq$ r.n.

Proof. It is the same as for theorem 3.2 of [6], using now theorem 1 . We recall that for $\varphi \in \mathcal{E}^{+}$and $\varphi_{m} \in \mathcal{C}, m \in \mathbb{N}$, as in lemma 1 , we will have $T^{0}(t) \varphi_{m}^{0}(\theta) \stackrel{m \rightarrow \infty}{\longrightarrow} T^{0}(t) \varphi^{0}(\theta)$ and then $T^{0}(t)_{\mid \mathcal{C}_{D^{0}}}=0 \Rightarrow T^{0}(t)=0$.

Remark 4. We have from [7] theorem 5.1 that if $V$ is any subset of the complex plane and $U=\left\{x \in \mathbb{R} \mid \exists\right.$ sequence $\left\{z_{k}\right\}_{k \in \mathbb{N}}, z_{k} \in V$ with $\operatorname{Re} z_{k} \rightarrow x$ and $\left|\operatorname{Im} z_{k}\right| \rightarrow \infty$ as $k \rightarrow \infty\}$, then for almost all real $t$, the inclusion $e^{t(U+i \mathbb{R})} \subset \overline{e^{t V}}$ holds, where $U+i \mathbb{R}=$ $\{z \in \mathbb{C} \mid \operatorname{Re} z \in U\}$. To see that this inclusion may not hold for all $t$, let us consider $V=\{0, \pm i, \pm 2 i, \pm 3 i, \cdots\}$ so $U=\{0\}, e^{t(i \mathbb{R})}$ is the unit circle for $t \neq 0$ and we note that $e^{t(i \mathbb{R})} \subset \overline{e^{t V}}$ if and only if $t / \pi$ is irrational.

Since det $H(\lambda)$ is an analytic almost periodic function of $\lambda$, we have from [6] lemma 3.2 that if $\operatorname{det} H(\lambda)=0$ for some $\lambda \in \mathbb{C}$, then there exists a sequence $\left\{\lambda_{k}\right\}_{k \in \mathbb{N}}$ such that $\operatorname{det} H\left(\lambda_{k}\right)=0,\left|\lambda_{k}\right| \rightarrow \infty$ and $\operatorname{Re} \lambda_{k} \rightarrow \operatorname{Re} \lambda$ as $k \rightarrow \infty$. If we take $V=\sigma\left(\mathbb{A}^{0}\right)=\{\lambda \in$ $\mathbb{C} \mid \operatorname{det} H(\lambda)=0\}$, then we have $U=\overline{\mathcal{Z}}$ and $e^{t(\overline{\mathcal{Z}}+i \mathbb{R})}=\left\{\mu|| \mu \mid=e^{\xi t}, \xi \in \overline{\mathcal{Z}}\right\}$ and from theorem 2 and the above result from [7], we conclude that

$$
\sigma\left(T^{0}(t)\right) \backslash\{0\}=\overline{e^{t \sigma\left(\mathbf{A}^{0}\right)}} \backslash\{0\}
$$

for almost all $t \geq 0$.

\section{The Neutral FDE}

Passing now to equation $(N)$ of section 2, we first generalize the lemma 4.1 of [6].

Lemma 1. For the equations $(N)$ and $(D)_{0}$ and their flows, given in section 2, we have: $T(t)-T^{0}(t) \circ \Psi: \mathcal{G}^{+} \rightarrow \mathcal{G}^{+}$is a compact operator for each $t \geq 0$, where $\Psi$ is the projection given in remark 1 of section 3 .

Proof. Analogous to the lemma 4.1 of [6]. Recall that $\mathcal{R}\left(I_{\mathcal{G}^{+}}-\Psi\right)$ has finite dimension.

We denote by $P \sigma(L), R \sigma(L)$ and $C \sigma(L)$ the point, the residual and the continuous parts of the spectrum of a linear operator $L$.

We generalize now the theorem 4.1 of [6].

Theorem 1. With the notation of section 2, for the flow of equation $(N)$, we have:

i) $P \sigma(T(t)) \backslash\{0\}=P \sigma(T(t) \mid \mathcal{C}) \backslash\{0\}=\left\{e^{\lambda t} \mid \operatorname{det} \Delta(\lambda)=0\right\}$

ii) $R \sigma(T(t)) \cup C \sigma(T(t)) \subset\left\{\mu|| \mu \mid=e^{\xi t}, \xi \in \overline{\mathcal{Z}}\right\} \cup\{0\}$ where $\mathcal{Z}$ is given in theorem

2 of section 3.

iii) $\sigma(T(t)) \backslash\{0\}=\overline{e^{t \sigma(A)}} \backslash\{0\}$ a.e. in $t \geq 0$. 
Proof. i) Suppose $t_{0}>0, \lambda_{0} \in \mathbb{C}$ and $\varphi \in \mathcal{G}^{+}$such that $T\left(t_{0}\right) \varphi=e^{\lambda_{0} t_{0}} \varphi \neq 0$.

We show that there is a $c \in \mathbb{E}^{n}$ such that $z(t)=c \cdot e^{\lambda t} \neq 0$ is the solution of $(N)$ with initial data $\psi(\theta)=c . e^{\lambda \theta}$, where $\lambda=\lambda_{0}+\frac{2 \pi i m}{t_{0}}$ for some $m \in \mathbb{N}$, that is; we find a continuous (in fact, exponential) eigenvector for the eigenvalue $e^{\lambda_{0} t_{0}}$ of $T\left(t_{0}\right)$.

Let $x_{t}=T(t) \varphi, t \geq 0$. The function $t \mapsto e^{-\lambda_{0} t} x(t)$ is periodic of period $t_{0}$ and then there is a $m \in \mathbb{N}$ such that the $m$-th Fourier coefficient is nonzero, that is,

$$
c=\frac{1}{t_{0}} \int_{0}^{t_{0}} e^{\frac{-2 \pi i m}{t_{0}} s} x(s) e^{-\lambda_{0} s} d s=\frac{1}{t_{0}} \int_{0}^{t_{0}} x(s) e^{-\lambda s} d s \neq 0
$$

and we have

$$
\begin{aligned}
c e^{\lambda(t+\theta)} & =\frac{1}{t_{0}} \int_{0}^{t_{0}} x(s) e^{\lambda(t+\theta-s)} d s \\
& =\frac{1}{t_{0}} \int_{0}^{t_{0}} x(t+u+\theta) e^{-\lambda u} d u \\
& =\frac{1}{t_{0}} \int_{0}^{t_{0}}\left[T(t) x_{u}\right](\theta) e^{-\lambda u} d u \\
& =\left[T(t)\left(\frac{1}{t_{0}} \int_{0}^{t_{0}} x_{u}(\cdot) e^{-\lambda u} d u\right)\right](\theta)=\left[T(t)\left(c \cdot e^{\lambda \cdot}\right)\right](\theta) .
\end{aligned}
$$

To prove the last equalities, let $\Delta$ be any partition of interval $\left[0, t_{0}\right]\left(0=u_{0}<u_{1} \cdots<\right.$ $\left.u_{k}=t_{0}, \bar{u}_{i} \in\left[u_{i-1}, u_{i}\right], \Delta u_{i}=u_{i}-u_{i-1}\right)$ and we have:

$$
\begin{aligned}
& {\left[T(t)\left(\frac{1}{t_{0}} \sum_{i=1}^{k} x_{\bar{u}_{i}}(\cdot) e^{-\lambda \bar{u}_{i}} \Delta u_{i}\right)\right](\theta)=\frac{1}{t_{0}} \sum_{i=1}^{k}\left[T(t) x_{\bar{u}_{i}}\right](\theta) e^{-\lambda \bar{u}_{i}} \Delta u_{i} \rightarrow} \\
& \stackrel{\|\Delta\| \rightarrow 0}{\longrightarrow} \frac{1}{t_{0}} \int_{0}^{t_{0}}\left[T(t) x_{u}\right](\theta) e^{-\lambda u} d u, \text { but } \\
& \left\|\left[T(t)\left(\frac{1}{t_{0}} \int_{0}^{t_{0}} x_{u}(\cdot) e^{-\lambda u} d u\right)\right](\theta)-\left[T(t)\left(\frac{1}{t_{0}} \sum_{i=1}^{k} x_{\overline{u_{i}}}(\cdot) e^{-\lambda \overline{u_{i}}} \Delta u_{i}\right)\right](\theta)\right\| \\
& \leq\|T(t)\| \cdot \frac{1}{t_{0}} \cdot\left\|\int_{0}^{t_{0}} x_{u}(\cdot) e^{-\lambda u} d u-\sum_{i=1}^{k} x_{\overline{u_{i}}}(\cdot) e^{-\lambda \bar{u}_{i}} \Delta u_{i}\right\|
\end{aligned}
$$




$$
\begin{aligned}
& =\|T(t)\| \frac{1}{t_{0}} \sup _{\bar{\theta} \in[-r, 0]}\left\|\int_{0}^{t_{0}} x_{u}(\bar{\theta}) e^{-\lambda u} d u-\sum_{i=1}^{k} x_{\overline{u_{i}}}(\bar{\theta}) e^{-\lambda \bar{u}_{i}} \Delta u_{i}\right\| \\
& \leq \underbrace{\|T(t)\| \frac{1}{t_{0}} \sup _{\bar{\theta} \in[-r, 0]}\left\|e^{\lambda \bar{\theta}}\right\|}_{K} \cdot \sup _{\bar{\theta} \in[-r, 0]} \| \int_{0}^{t_{0}} x(u+\bar{\theta}) e^{-\lambda(u+\bar{\theta})} d u \\
& -\sum_{i=1}^{k} x\left(\bar{u}_{i}+\bar{\theta}\right) e^{-\lambda\left(\bar{u}_{i}+\bar{\theta}\right)} \Delta u_{i} \| ! \\
& \leq K \cdot \sup _{\bar{\theta} \in[-r, 0]}\left\|\int_{\bar{\theta}}^{t_{0}+\bar{\theta}} x(s) e^{-\lambda s} d s-\sum_{i=1}^{k} x\left(\bar{u}_{i}+\bar{\theta}\right) e^{-\lambda\left(\overline{u_{i}}+\bar{\theta}\right)} \Delta u_{i}\right\|=K \cdot M(\Delta)
\end{aligned}
$$

Since the function $t \mapsto y(t) \stackrel{\text { def. }}{=} x(t) e^{-\lambda t}$ is periodic of period $t_{0}$, we have

$$
M(\Delta)=\sup _{\left.\bar{\theta} \in[-r, 0] \cap]-t_{0}, 0\right]}\left\|\int_{0}^{t_{0}} y(s) d s-\sum_{i=1}^{k} y\left(\bar{u}_{i}+\bar{\theta}\right) \Delta u_{i}\right\|
$$

For each $\left.\bar{\theta} \in[-r, 0] \cap]-t_{0}, 0\right]$, let us call $\Delta+\bar{\theta}$ the translation of $\Delta$ to the interval $\left[\bar{\theta}, t_{0}+\bar{\theta}\right]\left(\bar{\theta}=u_{0}+\bar{\theta}<u_{1}+\bar{\theta} \cdots<u_{k}+\bar{\theta}=t_{0}+\bar{\theta}\right)$. Define $l=l(\bar{\theta}) \in\{1,2, \cdots, k\}$ such that $u_{l-1}+\bar{\theta} \leq 0<u_{l}+\bar{\theta}$. We have the following situation (where we delete the first summation on the right hand side if $l=1$ and the last summation if $l=k$ ):

$$
\begin{aligned}
& \sum_{i=1}^{k} y\left(\bar{u}_{i}+\bar{\theta}\right) \Delta u_{i} \\
= & \sum_{i=1}^{l-1} y\left(\bar{u}_{i}+\bar{\theta}+t_{0}\right) \Delta u_{i}+y\left(\bar{u}_{l}+\bar{\theta}\right) \Delta u_{l}+\sum_{i=l+1}^{k} y\left(\bar{u}_{i}+\bar{\theta}\right) \Delta u_{i} \\
= & \sum_{j=k-l+2}^{k} y\left(\bar{s}_{j}(\bar{\theta})\right) \Delta s_{j}(\bar{\theta})+y\left(t_{0}\right) \underbrace{\left[t_{0}-\left(u_{l-1}+\bar{\theta}+t_{0}\right)\right]}_{\Delta s_{k+1}(\bar{\theta})}+y(0) \underbrace{\left[u_{l}+\bar{\theta}-0\right]}_{\Delta s_{1}(\bar{\theta})} \\
& +\left[y\left(\bar{u}_{l}+\bar{\theta}\right)-y(0)\right]\left[u_{l}-u_{l-1}\right]+\sum_{j=2}^{k-l+1} y\left(\bar{s}_{j}(\bar{\theta})\right) \Delta s_{j}(\bar{\theta}) \\
& k+1 \\
= & \sum_{j=1}^{k+1} y\left(\bar{s}_{j}(\bar{\theta})\right) \Delta s_{j}(\bar{\theta})+\left[y\left(\bar{u}_{l}+\bar{\theta}\right)-y(0)\right] \Delta u_{l} .
\end{aligned}
$$

This defines the partition $\Delta_{\bar{\theta}}$ of interval $\left[0, t_{0}\right]\left(0=s_{0}(\bar{\theta})<s_{1}(\bar{\theta}) \cdots<s_{k+1}(\bar{\theta})=\right.$ $\left.t_{0}, \bar{s}_{j}(\bar{\theta}) \in\left[s_{j-1}(\bar{\theta}), s_{j}(\bar{\theta})\right], \Delta s_{j}(\bar{\theta})=s_{j}(\bar{\theta})-s_{j-1}(\bar{\theta})\right)$ as shown in the figure 
Therefore

$$
M(\Delta) \leq \sup _{\left.\bar{\theta} \in[-r, 0] \cap]-t_{0}, 0\right]}\left\|\int_{0}^{t_{0}} y(s) d s-\sum_{j=1}^{k+1} y\left(\bar{s}_{j}(\bar{\theta})\right) \Delta s_{j}(\bar{\theta})\right\|+2 \sup _{s \in\left[0, t_{0}\right]}\|y(s)\| \cdot\|\Delta\| .
$$

Since $\left\|\Delta_{\bar{\theta}}\right\| \leq\|\Delta\|$ for any $\bar{\theta}$ in $[-r, 0]$, we see that $M(\Delta)$ goes to zero when $\|\Delta\| \rightarrow 0$. We have also $\frac{1}{t_{0}} \int_{0}^{t_{0}} x_{u}(\theta) e^{-\lambda u} d u=\frac{1}{t_{0}} \int_{0}^{t_{0}} x(u+\theta) e^{-\lambda(u+\theta)} d u \cdot e^{\lambda \theta}=c . e^{\lambda \theta}$.

By the same arguments as for theorem 4.1 of [6] we prove ii), using the result of Gohberg and Krein in the version of lemma 2 of [6] and using theorem 2 of section 3 and lemma 1.

For iii) we use theorem 5.1 of [7].

In the same way as theorem 4.2 of [6] we can show that:

Theorem 2. Suppose $\alpha \notin \overline{\operatorname{Re} \sigma(\mathbb{A})}$, i.e., det $\Delta(\lambda) \neq 0$ in some strip $\mid \operatorname{Re} \lambda-$ $\alpha \mid<\delta, \delta>0$. Then $\mathcal{G}^{+}=P \oplus Q$, where $P, Q$ are closed subspaces invariant under $T(t)$.

The restriction of the semigroup to $P$ may be extended to a group $\left\{T(t)_{\mid P}\right\}_{t \in \mathbb{R}}$ of isomorphisms of $P$. Finally, there exists a constant $M$ such that $\left\|T(t)_{\mid Q}\right\| \leq$ $M e^{(\alpha-\delta) t}$ for $t \geq 0$ and $\left\|T(t)_{\mid P}\right\| \leq M e^{(\alpha+\delta) t}$ for $t \leq 0$ (see also [3], chap.II, $\S 4$, theorems 3 and 4.)

We now study the corresponding decomposition for the nonhomogeneous equation

$$
\frac{d}{d t}\left(D x_{t}-H(t)\right)=L x_{t}
$$

for a given regulated right-continuous forcing function $H$.

For each $t, t_{0} \in \mathbb{R}, t \geq t_{0}$, we have a bounded linear operator $\mathcal{K}\left(t, t_{0}\right) \in$ $\mathcal{L}\left(G^{+}\left(\left[t_{0}, t\right], \mathbb{E}^{n}\right), \mathcal{G}^{+}\right)$such that the solution of $(N)_{H}$ for $t \geq t_{0}$ with $x_{t_{0}}=\varphi \in \mathcal{G}^{+}$is given by $x_{t}\left(t_{0}, \varphi, H\right)=T\left(t-t_{0}\right) \varphi+\mathcal{K}\left(t, t_{0}\right) H$, where $\{T(t)\}_{t \geq 0}$ is the flow of $(N)$ as in section 2 .

From [3] we have that $\mathcal{K}\left(t, t_{0}\right) H=\chi_{0} H(t)-T\left(t-t_{0}\right) \chi_{0} H\left(t_{0}\right)-\int_{t_{0}}^{t} d_{\sigma} T(t-\sigma) \chi_{0} H(\sigma)$ where, for $p \in \mathbb{E}^{n}$, we have $\chi_{0} p(\theta)=0$ for $-r \leq \theta<0$ and $\chi_{0} p(0)=p$ and $\left(\int_{t_{0}}^{t} d_{\sigma} T(t-\sigma) \chi_{0} H(\sigma)\right)(\theta)=\int_{t_{0}}^{t} d_{\sigma} X(t+\theta-\sigma) H(\sigma)$, the integral being in $\mathbb{E}^{n}$, and the fundamental matrix $X(t) \in \mathcal{L}\left(\mathbb{E}^{n}\right)$ given by $X(t) p=T(t) \chi_{0} p(0)$.

In [3] (see chap.II, $\S 4$, remark 7), we generalize the theorem 4.3 of [6] as a consequence of theorem 2 and the variation-of-constants formula for equation $(N)_{H}$. Then we have:

Theorem 3 Suppose $\alpha \notin \overline{\operatorname{Re} \sigma(\mathbb{A})}$ and $\mathcal{G}^{+}=P \oplus Q$ is the decomposition provided by theorem 2. We write $\varphi=\varphi^{P}+\varphi^{Q} \in P \oplus Q$. Then, there exist matrix-functions of bounded variation $X^{P}$ and $X^{Q}$, such that:

$$
\begin{aligned}
x_{t}^{Q}\left(t_{0}, \varphi, H\right)= & T\left(t-t_{0}\right) \varphi^{Q}+\left(\chi_{0} H(t)\right)^{Q}-T\left(t-t_{0}\right)\left(\chi_{0} H\left(t_{0}\right)\right)^{Q} \\
& -\int_{t_{0}}^{t} d_{\sigma} T(t-\sigma)\left[\chi_{0} H(\sigma)\right]^{Q}
\end{aligned}
$$


and

$$
\begin{aligned}
x_{t}^{P}\left(t_{0}, \varphi, H\right) & =T\left(t-t_{0}\right) \varphi^{P}+\left(\chi_{0} H(t)\right)^{P}-T\left(t-t_{0}\right)\left(\chi_{0} H\left(t_{0}\right)\right)^{P} \\
& -\int_{t_{0}}^{t} d_{\sigma} T(t-\sigma)\left[\chi_{0} H(\sigma)\right]^{P}
\end{aligned}
$$

for $t \geq t_{0}$, where

$$
\left(\int_{t_{0}}^{t} d_{\sigma} T(t-\sigma)\left[\chi_{0} H(\sigma)\right]^{P, Q}\right)(\theta)=\int_{t_{0}}^{t} d_{\sigma} X^{P, Q}(t+\theta-\sigma) H(\sigma)
$$

for $\theta \in[-r, 0]$.

Remark 2. The matrix functions $X^{Q}$ and $X^{P}$ are the same as in theorem 4.3 of [6] (our $X^{P, Q}(t+\theta)$ are his $T(t) \chi_{0}^{P, Q}(\theta)$ ) and we obtain the estimates given in that theorem.

\section{References}

[1] O. Diekmann, Perturbed Dual Semigroups and Delay Equations, Dynamics of Infinite Dimensional Systems (Lisbon 1986), 67-73. NATO Adv. Sci. Inst. Série F vol. 37, edited by Chow, S. N. and Hale, J. K.-Springer Berlin-New York, 1987.

[2] L. Fichmann, Equações integrais de Volterra-Stieltjes e Equações do tipo neutro, Tese de mestrado, IME-USP, Brasil, 1984.

[3] L.Fichmann, Equações Diferenciais Neutras com condições iniciais descontinuas, Tese de doutorado, IME-USP, Brasil, 1991.

[4] J. Hale, Theory of Functional Differential Equations, Springer-Verlag, 1977.

[5] D. Henry, "Small solutions of linear autonomous FDEs," J. Differential Equations 8 (1970).

[6] D. Henry, "Linear Autonomous Neutral Functional Differential Equations," Journal of Differential Equations 15 (106-128), 1974.

[7] D. Henry, "A property of the exponential function," Publ .Mat, UAB (Universitat Autônoma de Barcelona), vol.31, no 1, artigo 5-Bellaterra, abril 1987.

[8] C.S. Hönig, Volterra-Stieltjes Integral Equations: Functional Analytic Methods, Linear Constraints, Amsterdam, North-Holland, 1975 (North-Holland Math. Studies, 16).

[9] C.S. Hönig, Equations Integrales generalisées et aplications, Probl. Mathématiques d'Orsay, Exposé no $5,1981-82$.

[10] J.C.F. Oliveira and L. Fichmann, "Discontinuous solutions of Neutral Functional Differential Equations," Publ. Mat. UAB (Universitat Autonoma de Barcelona) Vol. 37, no.2, (369-386), 1993, Bella.terra.

[11] S. Schwabick, "A survey of some new results for regulated functions," 280 Seminário Brasileiro de Análise, 1988, SBM, p.201-209.

Departamento de Matemática - IME-Universidade de São Paulo Caixa Postal 20570 - 01452-990 - São Paulo - SP - Brasil. 